'Pontificia Universidad Católica de Chile. Santiago, Chile. 2Departamento de Dermatología, Pontificia Universidad Católica de Chile. Santiago, Chile.

${ }^{3}$ Departamento de Anatomía Patológica, Pontificia Universidad Católica de Chile. Santiago, Chile.

Los autores declaran no tener apoyo de fuentes de financiación externas.

Los autores declaran no tener conflictos de interés.

Recibido el 7 de noviembre de 2017, aceptado el 24 de julio de 2018.

Correspondencia a: Montserrat Molgó N. Departamento de Dermatología, Pontificia Universidad Católica de Chile.

Avda. Vicuña Mackenna 4686, Macul. Santiago, Chile. montsemolgo1@gmail.com

\section{Sospecha diagnóstica de síndrome de Ehlers Danlos tipo vascular: reporte de un caso y revisión de literatura}

\author{
CAROLINA CEVALLOS B. ${ }^{1}$, EMILIO VARGAS ${ }^{2}$, \\ SERGIO GONZÁLEZ B. ${ }^{3}$, MONTSERRAT MOLGÓ ${ }^{2}$
}

\section{Suspected vascular ehlers danlos syndrome. Case report}

Ehlers Danlos Syndrome comprises a heterogeneous group of genetic disorders of the connective tissue, due to defects in collagen or its modifying enzymes. We report a 21 years old male presenting with translucent skin revealing the subcutaneous venous pattern. He had a thin face, large-appearing eyes, thin lips, thin nose, joint hypermotility and history of hip dysplasia. A vascular Ehlers Danlos Syndrome was suspected. However, the genetic study to confirm the diagnosis was not done.

(Rev Med Chile 2018; 146: 938-942)

Key words: Connective Tissue Diseases; Ehlers-Danlos Syndrome; Genetic Diseases, Inborn.
1 síndrome de Ehlers Danlos (SED) comprende un grupo heterogéneo de enfermedades hereditarias del tejido conectivo con gran heterogeneidad genética y clínica. Debe sospecharse en todo paciente que presente hiperlaxitud cutánea, articular y fragilidad tisular. Su incidencia aproximada es de 1 en 5.000, siendo el tipo hipermóvil el más común, seguido por los tipos clásico y vascular ${ }^{1}$.

En su fisiopatología participarían alteraciones hereditarias en genes responsables de la síntesis y procesamiento de diferentes formas de colágeno, proteínas responsables de dar estructura al tejido conectivo, constituyente de tejidos y órganos como la piel, tendones, ligamentos, músculos, vasos sanguíneos, esqueleto y ojos ${ }^{2,3}$.

Desde 1998 para el diagnóstico de SED se utilizó la clasificación nosológica de Villefranche que distinguía 6 tipos, pero desde el 2017 se adopta la nueva Clasificación internacional para SED que mantiene nombres descriptivos, criterios clínicos mayores y menores y agrega la confirmación diagnóstica mediante la identificación de la variante genética específica, excepto en el caso del SED hipermóvil, tipo en que aún no se ha identificado el(los) genes responsables. La importancia de un diagnóstico preciso de la variante genética radica en su capacidad de distinguir entre tipos con superposición clínica, en la información que entrega en relación a pronóstico, manejo específico, patrón de herencia y formación de cohortes precisas de pacientes para investigación de la enfermedad ${ }^{4}$.

\section{Caso clínico}

Paciente masculino de 21 años, consulta en Dermatología por marcada trama vascular en espalda. Al examen físico fascies peculiar con nariz fina, labios delgados, mejillas hundidas y ojos prominentes, hiperlaxitud articular, especialmente de articulaciones pequeñas con score de Beighton de 9 puntos (Figura la y 1b). Piel extremadamente laxa, fina y traslúcida que a nivel de la espalda y pecho permite identificar con claridad la trama vascular venosa subyacente (Figura 1c). En su 

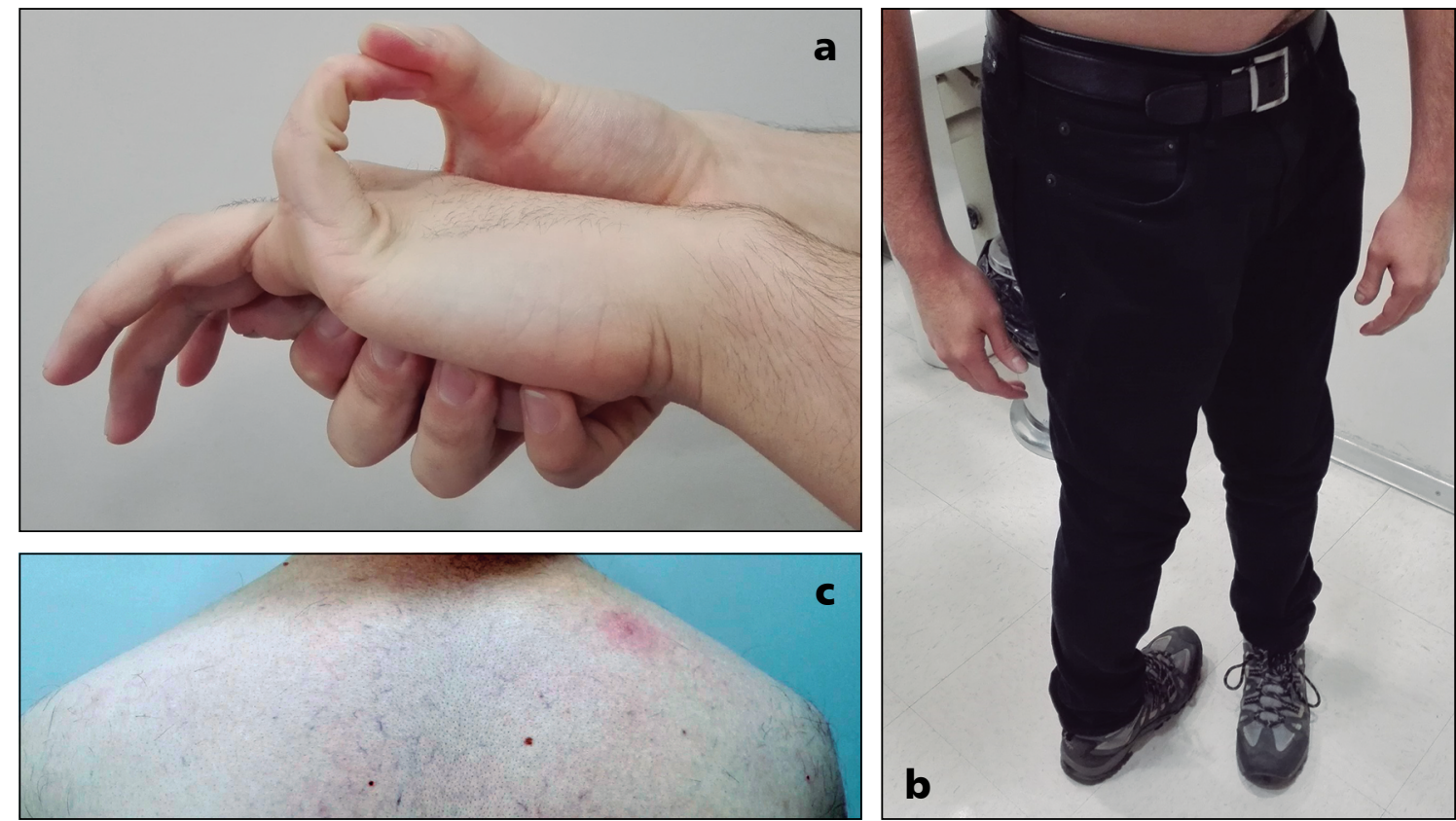

Figura 1. Alteraciones del examen físico sugerentes de vSED. (a) Hipermovilidad de pequeñas articulaciones, con dorsiflexión pasiva de quinto metacarpiano mayor a $90^{\circ}$. (b) Hipermovilidad de grandes articulaciones con compromiso de articulación coxofemoral. (c) En la región dorsal del paciente se aprecia una piel fina y traslúcida con visibilidad de la trama vascular.

historia es relevante el antecedente de displasia de cadera en la infancia, equimosis recurrentes no siempre relacionadas a trauma, episodios de cefalea con aura, acompañada de hipostesia en el brazo izquierdo y sobretodo, el antecedente familiar de muerte precoz de dos tíos maternos por accidente vascular. Dada la sospecha clínica de SED tipo vascular (vSED) versus tipo hipermóvil, es evaluado por genetista, quien coincide con la sospecha de vSED, y deriva para estudio vascular (electrocardiograma, ecocardiografía y angioresonancia) y genético con panel de aortopatía Invitae (para estudio de variantes genéticas de COL3A1, entre otros), los cuales no fueron realizados por el gran coste económico implicado. Además el paciente es evaluado en traumatología para tratamiento de dolor articular crónico.

\section{Discusión}

Se debe sospechar SED en pacientes con hiperlaxitud articular, cutánea y fragilidad tisular. Estos elementos pueden presentarse en la historia del paciente como múltiples luxaciones y esguinces, cicatrización anómala, dehiscencia de heridas quirúrgicas, aparición de equimosis y hematomas de forma espontánea o ante traumas mínimos y en algunos casos de enfermedad severa, como historia de ruptura vascular o visceral espontánea. En la exploración física se presentaran como hipermovilidad articular, que puede objetivarse mediante el score de Beighton ${ }^{4}$ y mútiples elementos cutáneos que hacen la patología reconocible para el dermatólogo entrenado, entre ellos: hiperlaxitud de la piel, extensibilidad de lóbulos auriculares, marcada movilidad lingual que en muchos casos permite topar la punta de la nariz, estrías en pacientes sin un factor claro predisponente, cicatrices atróficas, en papel de cigarrillo o moluscoides, llamativa trasluscencia cutánea que permite visualizar con claridad los vasos venosos subyacentes ${ }^{5}$. El diagnóstico de la variante vascular, que es la más severa y de peor pronóstico, debe ser considerado en pacientes jóvenes que además han presentado espontáneamente disección de un vaso de gran calibre o ruptura visceral. Ante la sospecha de SED debe realizarse evaluación genética para la 
confirmación diagnóstica ${ }^{4}$.

Por su severidad se debe conocer reconocer el SED tipo vascular (vSED) que corresponde a la variante de herencia autosómica dominante debida a mutación del gen COL3A1, que codifica las cadenas de colágeno tipo III $^{6-8}$. El colágeno tipo III es la principal proteína en las paredes de los vasos sanguíneos y órganos huecos, lo cual explica en quienes presentan la mutación, el aumento de equímosis, la fragilidad arterial e intestinal y uterina. El espectro clínico se explica en parte, por la heterogeneidad alélica. Los criterios clínicos actuales de muestran en la Tabla 1.

De acuerdo con la nueva clasificación, los criterios mínimos sugerentes de vSED son la presencia de historia familiar, ruptura o disección arterial en individuos menores de 40 años de edad, ruptura inexplicada de colon sigmoides o neumotórax espontáneo en presencia de otras características consistentes con vSED. Estos individuos deben someterse a estudios diagnósticos para confirmación ${ }^{4}$.

Los hallazgos histológicos, si bien no forman parte de los criterios diagnósticos, pueden ser útiles en pacientes en los cuales el estudio genético no sea posible de realizar. En la microscopía óptica se puede observar una dermis adelgazada, dentro de la cual las fibras colágenas son más escasas o irregulares ${ }^{9}$. La microscopía electrónica es normal en la mayoría de los pacientes, aunque en algunos casos es posible observar fibras de colágeno dispersas, pequeñas de diámetro irregular y fibras elásticas abundantes, pero cortas y fragmentadas (Figura 2). También es posible observar fibroblastos con un retículo endoplásmico rugoso, cisternas dilatadas y un material proteico en su interior que corresponde a moléculas de procolágeno tipo $\mathrm{II}^{10}$.

En los pacientes con vSED la mayoría de los eventos arteriales sintomáticos son disecciones, que en general son autolimitadas y pueden no requerir intervención invasiva. En caso de ruptura arterial se requiere reparación urgente. Existe riesgo aumentado de disección de arterias coronarias y en consecuencia, de infarto agudo al miocardio. Respecto a la vigilancia, no existe consenso sobre la necesidad de estudios vasculares y en algunos casos se opta por estudio angiográfico por Scanner o Resonancia Magnética con frecuencia anual, sin embargo tampoco se han establecido criterios para realizar intervenciones.

\section{Tabla 1. Criterios clínicos para vEDS según la Clasificación Internacional para EDS 2017}

\section{A. Criterios mayores}

1. Antecedentes familiares de vEDS con una variante causal documentada en COL3A1

2. Ruptura arterial a una edad temprana

3. Perforación espontánea del colon sigmoides en ausencia de enfermedad diverticular conocida u otra patología intestinal

4. Ruptura uterina durante el tercer trimestre en ausencia de cesáreas previas y/o laceraciones perineales periparto

5. Formación de fístula del seno carotideo-cavernoso (CCSF) en ausencia de trauma

\section{B. Criterios menores}

1. Equimosis no relacionados con trauma $y / 0$ en sitios inusuales como las mejillas y espalda

2. Piel delgada, translúcida, con mayor visibilidad venosa

3. Apariencia facial característica

4. Neumotórax espontáneo

5. Acrogeria

6. Pies equinovaro

7. Displasia congénita de cadera

8. Hipermovilidad de pequeñas articulaciones

9. Rotura muscular y de tendones

10. Queratocono

11. Recesión gingival y fragilidad gingival

12. Venas varicosas de inicio temprano (menores de 30 años, nulíparas en mujeres)

Adaptada al español desde: Malfait F, Francomano C, Byers P, Belmont J, Berglund B, Black J, et al. The 2017 international classification of the Ehlers-Danlos syndromes. Am J Med Genet C Semin Med Genet. 2017;175(1):8-26.

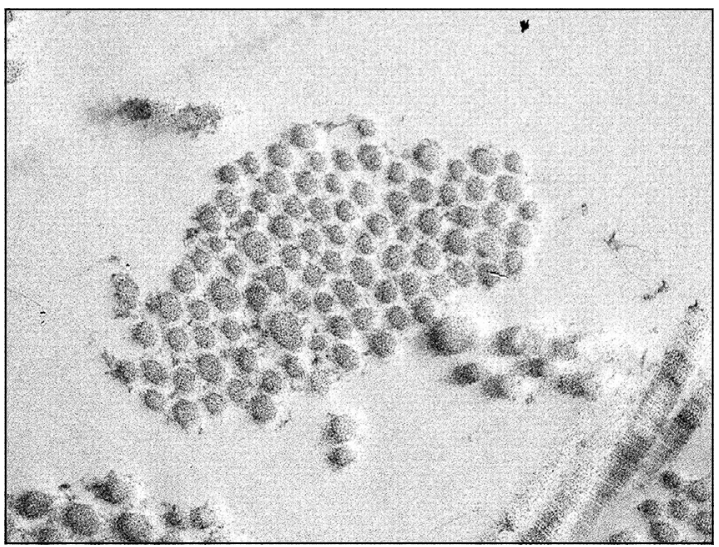

Figura 2. Hallazgos ultraestructurales sugerentes de SED: Microscopía electrónica de transmisión que muestra fibras de colágeno en corte transversal con variaciones manifiestas en el diámetro. Citrato de plomo-acetato de uranilo, aumento original de 43.000. (Cortesía del Dr. Sergio González). 
La complicación gastrointestinal más común es la ruptura espontánea de colon, usualmente sigmoides ${ }^{7,8}$, cuyo tratamiento usualmente es colostomía.

La insuficiencia venosa superficial es más prevalente en estos pacientes comparado con población general $(37 \% \text { vs } 17-23 \%)^{8}$, además de su aparición a edades más precoces (44\% de los pacientes diagnosticados antes de los 20 años $)^{11}$.

La translucencia de la piel con visibilidad del patrón venoso es una de las características típicas descritas en vSED. La formación de hematomas puede aumentar con uso de Aspirina o AINEs y aún más con la terapia anticoagulante. La acrogeria es poco común, así también la presencia de alopecia, aunque puede llegar a ser llamativa en algunas mujeres.

Actualmente, la esperanza de vida promedio de los individuos afectados con vSED es de 51 años (rango entre los 10 a 80 años) ${ }^{7,8}$. La principal causa de mortalidad es la disección o ruptura arterial con falla orgánica. Es por eso que el objetivo del manejo médico es minimizar la probabilidad de eventos adversos y asegurar que la calidad de vida sea mínimamente afectada. Con respecto a las actividades deportivas, se desaconsejan los deportes de contacto y de gran intensidad, para prevenir disección o ruptura vascular. El principal objetivo de las intervenciones médicas ha sido mantener un control adecuado de presión arterial, en valor normal o límite inferior y prevenir alzas bruscas ${ }^{12}$. Las opciones farmacológicas son variadas, aunque actualmente existe sólo un estudio clínico con Celiprolol que demuestra que su uso permite retardar la aparición de eventos vasculares si se compara con pacientes no tratados, aunque el estudio presenta algunas fallas en su metodología ${ }^{13}$.

En líneas generales, el diagnóstico diferencial de un tipo de SED se debe realizar con alguna otra variante de la enfermedad y con otras enfermedades hereditarias del tejido conectivo como el Síndrome de Marfan, Loeys-Dietz, Larsen, Cutis laxa, Osteogénesis imperfecta y Síndrome de tortuosidad arterial ${ }^{14}$.

Es posible concluir entonces que el SED es una enfermedad infrecuente, poco conocida por médicos no especialistas, lo que dificulta su diagnóstico. Debe sospecharse en pacientes con hiperlaxitud articular, cutánea y fragilidad tisular. La variante vascular (vSED) es la más severa y de peor pronós- tico, debe tenerse presente en individuos jóvenes con elementos de SED que espontáneamente han presentado disección de un vaso de gran calibre o ruptura visceral o tienen antecedentes familiares de eventos de ese tipo en parientes jóvenes. La nueva clasificación publicada, permite un diagnóstico de precisión genética para un adecuado manejo, consejería y estudio familiar.

\section{Referencias}

1. Steinmann B, Royce PM, Superti-Furga A. The Ehlers-Danlos Syndrome, in Connective Tissue and Its Heritable Disorders: Molecular, Genetic, and Medical Aspects, Second Edition (eds P. M. Royce and B. Steinmann), John Wiley \& Sons, Inc., Hoboken, NJ, USA. doi: 10.1002/0471221929.ch9. 2002.

2. Callewaert B, Malfait F, Loeys B, De Paepe A. Ehlers-Danlos syndromes and Marfan syndrome. Best Pract Res Clin Rheumatol 2008; 22 (1): 165-89.

3. De Paepe A, Malfait F. The Ehlers-Danlos syndrome, a disorder with many faces. Clin Genet 2012; 82 (1): 1-11.

4. Malfait F, Francomano C, Byers P, Belmont J, Berglund $\mathrm{B}$, Black J, et al. The 2017 international classification of the Ehlers-Danlos syndromes. Am J Med Genet C Semin Med Genet 2017; 175 (1): 8-26.

5. Proske S, Hartschuh W, Enk A, Hausser I. [Ehlers-Danlos syndrome-20 years experience with diagnosis and classification at the university skin clinic of Heidelberg]. Journal der Deutschen Dermatologischen Gesellschaft = Journal of the German Society of Dermatology : JDDG 2006; 4 (4): 308-18.

6. Pope FM, Martin GR, Lichtenstein JR, Penttinen R, Gerson B, Rowe DW, et al. Patients with Ehlers-Danlos syndrome type IV lack type III collagen. Proc Natl Acad Sci USA 1975; 72 (4): 1314-6.

7. Pepin MG, Schwarze U, Rice KM, Liu M, Leistritz D, Byers $\mathrm{PH}$. Survival is affected by mutation type and molecular mechanism in vascular Ehlers-Danlos syndrome (EDS type IV). Genet Med 2014; 16 (12): 881-8.

8. Frank M, Albuisson J, Ranque B, Golmard L, Mazzella JM, Bal-Theoleyre L, et al. The type of variants at the COL3Al gene associates with the phenotype and severity of vascular Ehlers-Danlos syndrome. Eur J Hum Genet 2015; 23 (12): 1657-64.

9. Germain DP, Herrera-Guzmán Y. Vascular Ehlers-Danlos syndrome. Ann Genet 2004; 47 (1): 1-9.

10. Miranda GA, Frías AG, Hierro OS. Hiperelasticidad cutánea e hiperlaxitud articular. Problemas no clasificados. Dermatol Rev Mex 2008; 52 (3): 111-20.

11. Frank M, Says J, Denarie N, Messas E. Natural history of 
superficial venous insufficiency in patientswith vascular Ehlers-Danlos syndrome. Phleb Ann Vasc 2015; 68: 3440.

12. Byers PH, Belmont J, Black J, De Backer J, Frank M, Jeunemaitre $\mathrm{X}$, et al. Diagnosis, natural history, and management in vascular Ehlers-Danlos syndrome. Am J Med Genet C Semin Med Genet 2017; 175 (1): 40-7.

13. Ong K-T, Perdu J, De Backer J, Bozec E, Collignon P, Emmerich J, et al. Effect of celiprolol on prevention of cardiovascular events in vascular Ehlers-Danlos syndrome: a prospective randomised, open, blinded-endpoints trial. The Lancet 376 (9751): 1476-84.

14. Colombi M, Dordoni C, Chiarelli N, Ritelli M. Differential diagnosis and diagnostic flow chart of joint hypermobility syndrome/ehlers-danlos syndrome hypermobility type compared to other heritable connective tissue disorders. Am J Med Genet C Semin Med Genet 2015; 169C (1): 6-22. 